\title{
Crescentic Glomerulonephritis
}

National Cancer Institute

\section{Source}

National Cancer Institute. Crescentic Glomerulonephritis. NCI Thesaurus. Code C35444.

A histopathologic term for a pattern of diseases characterized by extensive crescent formation in the glomeruli; patients present clinically with rapid deterioration of renal function, and possible progression to end-stage renal failure within weeks or months. 